\title{
A simple method of establishing relations between Theta-functions of zero argument
}

By J. P. Datton, University of the Witwatersrand, Johannesburg, South Africa.

(Received. 31st March, 1933. Read 5th May, 1933.)

In connection with their proof of the fundamental relation ${ }^{1}$

$(0, \tau)$ :

$\vartheta_{1}^{\prime}=\vartheta_{2} \vartheta_{3} \vartheta_{4}$

Whittaker and Watson state ${ }^{2}$ that "several proofs of this important proposition have been given, but none are simple."

The following proof lays no claim to elegance; but it is simple in the sense that it follows naturally, by elementary processes, from the fundamental properties of the $\vartheta$-functions; having served their purpose in defining the functions, in determining their periodicity factors, and, in particular, in establishing the equation

$(v, \tau)$ :

$$
\frac{\partial \vartheta}{\partial \tau}=\frac{\pi}{4 i} \frac{\partial^{2} \vartheta}{\partial v^{2}},
$$

infinite series disappear completely.

A consideration of the periodicity factors, in conjunction with Liouville's theorem, leads to the relations ${ }^{3}$

$(v, \tau):$

$$
\frac{\partial}{\partial v}\left[\frac{\vartheta_{a}}{\vartheta_{\beta}}\right]=\phi(\tau) \frac{\vartheta_{\gamma} \vartheta_{\delta}}{\vartheta_{\beta}^{2}},
$$

where $\alpha, \beta, \gamma, \delta$ is any permutation of $1,2,3,4$.

The ultimate form of $\phi(\tau)$ depends upon (1), but without assuming that relation we find

$(0, \tau)$ :

$$
\phi(\tau)=\epsilon \frac{\vartheta_{1}^{\prime} \vartheta_{p}}{\vartheta_{q} \vartheta_{r}}, \quad \epsilon= \pm 1
$$

If $a=1$, then $\epsilon=+1$ and $p=\beta$; if $\beta=1, \epsilon=-1$ and $p=\alpha$; if $\gamma=1, \epsilon=\operatorname{sgn}(\alpha-\beta)$ and $p=\delta$.

1 For convenience, argument and parameter to be attached to each vacant Theta symbol are placed in front of each equation. Accents invariably indicate differentiation with respect to the argument $v$.

2 Whittaker and Watson: Modern Analysis (Cambridge, 1920), 470, footnote.

3 Whittaker and Watson : loc. cit., 477. 
We now differentiate equation (3) twice with respect to argument $v$, once with respect to parameter $\tau$. After the $\tau$ differentiation the relation (2) is applied. These operations yield, for all values of $v$,

$$
\begin{aligned}
& \vartheta_{\beta} \vartheta_{a}^{\prime}-\vartheta_{\beta}^{\prime} \vartheta_{\alpha}=\phi(\tau) \vartheta_{\gamma} \vartheta_{\delta} \\
& \vartheta_{\beta} \vartheta_{a}^{\prime \prime}-\vartheta_{\beta}^{\prime \prime} \vartheta_{a}=\phi(\tau)\left(\vartheta_{\gamma} \vartheta_{\delta}^{\prime}+\vartheta_{\gamma}^{\prime} \vartheta_{\delta}\right) \\
& \vartheta_{\beta} \vartheta_{a}^{\prime \prime \prime}+\vartheta_{\beta}^{\prime} \vartheta_{a}^{\prime \prime}-\vartheta_{\beta}^{\prime \prime} \vartheta_{a}^{\prime}-\vartheta_{\beta}^{\prime \prime \prime} \vartheta_{a}=\phi(\tau)\left(\vartheta_{\gamma} \vartheta_{\delta}^{\prime \prime}+2 \vartheta_{\gamma}^{\prime} \vartheta_{\delta}^{\prime}+\vartheta_{\gamma}^{\prime \prime} \vartheta_{\delta}\right) \\
& \vartheta_{\beta} \vartheta_{a}^{\prime \prime \prime}+\vartheta_{\beta}^{\prime \prime} \vartheta_{a}^{\prime}-\vartheta_{\beta}^{\prime} \vartheta_{a}^{\prime \prime}-\vartheta_{\beta}^{\prime \prime \prime} \vartheta_{a} \\
& =\phi(\tau)\left(\vartheta_{\gamma} \vartheta_{\delta}^{\prime \prime}+\vartheta_{\gamma}^{\prime \prime} \vartheta_{\delta}\right)+\pi^{-1} 4 i \vartheta_{\gamma} \vartheta_{\delta} \frac{d \phi(\tau)}{d \tau}
\end{aligned}
$$

Subtract (6) from (7), eliminate $\vartheta_{a}^{\prime \prime}$ by means of (5), simplify by introducing $\left(3^{\prime}\right)$. The result is

$$
(v, \tau): \quad \pi^{-1} 2 i \frac{d}{d \tau} \log \phi(\tau)=\frac{\vartheta_{\beta}^{\prime \prime}}{\vartheta_{\beta}}+\frac{\vartheta_{\gamma}^{\prime} \vartheta_{\delta}^{\prime}}{\vartheta_{\gamma} \vartheta_{\delta}}-\frac{\vartheta_{\beta}^{\prime} \vartheta_{\delta}^{\prime}}{\vartheta_{\beta}^{\prime} \vartheta_{\delta}}-\frac{\vartheta_{\beta}^{\prime} \vartheta_{\gamma}^{\prime}}{\vartheta_{\beta}^{\prime} \vartheta_{\gamma}}
$$

The right hand member is therefore independent of $v$, a fact that leads to tolerably complicated relations between the general functions which, fortunately, are not germane to the present discussion.

The equation (8) suggests two cases for special consideration according as the eliminated subscript $a$ is, or is not, unity.

Putting $a=1$ and $v=0$ in (8), we find

$$
(0, \tau): \quad \pi^{-1} 2 i \frac{d}{d \tau} \log \phi(\tau)=\vartheta_{\beta}^{-1} \vartheta_{\beta}^{\prime \prime}=\pi^{-1} 4 i \vartheta_{\beta}^{-1} \frac{d}{d \tau} \vartheta_{\beta},
$$

and therefore by (4)

$$
(0, \tau): \quad \frac{\vartheta_{1}^{\prime} \vartheta_{\beta}}{\vartheta_{\gamma} \vartheta_{\delta}}=\phi(\tau)=\vartheta_{\beta}^{2}+C_{\beta} .
$$

One could show that the constant of integration vanishes by making $q \rightarrow 0$, but it is more in harmony with the spirit of the present method to avoid limiting processes with infinite series. It follows from (10) that

$$
(0, \tau): \quad C_{2} \vartheta_{3}^{2} \vartheta_{4}^{2}=C_{3} \vartheta_{4}^{2} \vartheta_{2}^{2}=C_{4} \vartheta_{2}^{2} \vartheta_{3}^{2}
$$

for all values of $\tau$. Hence $C_{2}=C_{3}=C_{4}=0$.

Inserting this value of $C_{\beta}$, (10) becomes

$(0, \tau):$

$$
\vartheta_{1}^{\prime}=\vartheta_{2} \vartheta_{3} \vartheta_{4}
$$


Equation (9) may be written in the form

$$
(0, \tau): \quad \frac{1}{\vartheta_{1}{ }^{\prime}} \frac{d \vartheta_{1}{ }^{\prime}}{d \tau}+\frac{1}{\vartheta_{\beta}} \frac{d \vartheta_{\beta}}{d \tau}-\frac{1}{\vartheta_{\gamma}} \frac{d \vartheta_{\gamma}}{d \tau}-\frac{1}{\vartheta_{\delta}} \frac{d \vartheta_{\delta}}{d \tau}=\frac{\pi}{2 i} \frac{\vartheta_{\beta}{ }^{\prime \prime}}{\vartheta_{\beta}},
$$

and hence, on account of (2), we get the well-known relation

$$
(0, \tau): \quad \frac{\vartheta_{1}{ }^{\prime \prime \prime}}{\vartheta_{1}{ }^{\prime}}=\sum_{2}^{4} \frac{\vartheta_{\mu}{ }^{\prime \prime}}{\vartheta_{\mu}} \text {. }
$$

Consider next the case where the differentiated ratio in (3) does not contain $\vartheta_{1}$. Let

$(0, \tau): \quad \gamma=1 ; \quad \phi(\tau)=\epsilon \frac{\vartheta_{1}^{\prime} \vartheta_{\delta}}{\vartheta_{a} \vartheta_{\beta}} ; \quad \epsilon=\operatorname{sgn}(\alpha-\beta)$.

Then from (8) we have

$$
(v, \tau): \quad \pi^{-1} 2 i \frac{d}{d \tau} \log \phi(\tau)=\frac{\vartheta_{\beta}^{\prime \prime}}{\vartheta_{\beta}^{\prime}}-\frac{\vartheta_{\beta}^{\prime} \vartheta_{\delta}^{\prime}}{\vartheta_{\beta} \vartheta_{\delta}}+\frac{\vartheta_{1}^{\prime}}{\vartheta_{1}}\left(\frac{\vartheta_{\delta}^{\prime}}{\vartheta_{\delta}}-\frac{\vartheta_{\beta}{ }^{\prime}}{\vartheta_{\beta}}\right) .
$$

But, when $v=0$, we have

so that

$$
\begin{aligned}
\frac{d}{d \tau} \log \phi(\tau) & =\frac{d}{d \tau} \log \frac{\vartheta_{1}^{\prime} \vartheta_{\delta}}{\vartheta_{\beta} \vartheta_{a}} \\
& =\frac{\pi}{4 i}\left[\frac{\vartheta_{1}^{\prime \prime \prime}}{\vartheta_{1}^{\prime \prime}}+\frac{\vartheta_{\delta}^{\prime \prime}}{\vartheta_{\delta}}-\frac{\vartheta_{a}^{\prime \prime}}{\vartheta_{a}}-\frac{\vartheta_{\beta}^{\prime \prime}}{\vartheta_{\beta}}\right] \\
& =\frac{\pi}{2 i} \frac{\vartheta_{\delta}^{\prime \prime}}{\vartheta_{\delta}^{\prime}} \text { by (12), }
\end{aligned}
$$

$$
(v, \tau): \quad \frac{\vartheta_{\beta}^{\prime \prime}}{\vartheta_{\beta}}-\frac{\vartheta_{\beta}{ }^{\prime} \vartheta_{\delta}^{\prime}}{\vartheta_{\beta} \vartheta_{\delta}}+\frac{\vartheta_{1}{ }^{\prime}}{\vartheta_{1}}\left(\frac{\vartheta_{\delta}{ }^{\prime}}{\vartheta_{\delta}}-\frac{\vartheta_{\beta}{ }^{\prime}}{\vartheta_{\beta}}\right)=\frac{\vartheta_{\delta}^{\prime \prime}(0, \tau)}{\vartheta_{\delta}(0, \tau)} \text {. }
$$

For $v \rightarrow 0$, the indeterminate form on the left is easily evaluated by means of $\left(3^{\prime}\right)$, to give

$$
(0, \tau): \quad \frac{\vartheta_{\delta}^{\prime \prime}}{\vartheta_{\delta}}-\frac{\vartheta_{\beta}^{\prime \prime}}{\vartheta_{\beta}}=\frac{\vartheta_{1}^{2 \prime} \vartheta_{a}^{2}}{\vartheta_{\beta}^{2} \vartheta_{\delta}^{2}}
$$

when $\delta>\beta$.

Finally, uniting the three equations (16), we obtain

$$
(0, \tau): \quad \vartheta_{3}^{4}=\vartheta_{2}^{4}+\vartheta_{4}^{4} \text {. }
$$

This completes the deduction of the standard relations between Theta-functions of zero argument. 